\title{
Covariant gauge-natural conservation laws
}

\author{
M. Palese and E. Winterroth* \\ Department of Mathematics, University of Torino \\ via C. Alberto 10, 10123 Torino, Italy \\ e-mails: PALESE@DM.UNITO.IT, EKKEHART@DM.UNITO.IT
}

\begin{abstract}
When a gauge-natural invariant variational principle is assigned, to determine canonical covariant conservation laws, the vertical part of gaugenatural lifts of infinitesimal principal automorphisms - defining infinitesimal variations of sections of gauge-natural bundles - must satisfy generalized Jacobi equations for the gauge-natural invariant Lagrangian. Vice versa all vertical parts of gauge-natural lifts of infinitesimal principal automorphisms which are in the kernel of generalized Jacobi morphisms are generators of canonical covariant currents and superpotentials. In particular, only a few gauge-natural lifts can be considered as canonical generators of covariant gauge-natural physical charges.
\end{abstract}

2000 MSC: 58A20,58A32,58E30,58E40,58J10,58J70.

Key words: gauge-natural bundles, invariant variational principles, variational sequences, generalized Jacobi morphisms, conservation laws.

\section{Introduction}

In [1, 2] the general program was started of defining covariant conservation laws for field theories as generators of infinitesimal transformations of the basis manifold. In the classical Lagrangian formulation of field theories the description of symmetries and conserved quantities amounts to define suitable (vector) densities which generate the conserved currents; in all relevant physical theories this densities are found to be the divergence of skew-symmetric (tensor) densities, which are called superpotentials for the conserved currents. It is also well known that the importance of superpotentials relies on the fact that they can be integrated to provide conserved quantities associated with the conserved currents via the Stokes theorem (see e.g. [7] and references quoted therein). Within such a procedure, the generalized Bianchi identities for geometric field theories - introduced by Bergman to get (after an integration by parts procedure) a consistent equation between (local) divergences within the first variation formula - are in fact necessary and (locally) sufficient conditions for the conserved current $\epsilon$ to

\footnotetext{
${ }^{*}$ Both of them supported by GNFM of INdAM and University of Torino.
} 
be not only closed but also the divergence of a skew-symmetric (tensor) density (a superpotential) along solutions of the Euler-Lagrange equations. However, to "covariantize" such a derivation of Bianchi identities and superpotentials, background metrics or (fibered) connections have to be fixed a priori (see e.g. [4, 6, 7] and the wide literature quoted therein). The outcoming of such an ad hoc procedure is, notably, a triviality result concerning existence of global superpotentials for gauge-natural field theories despite of the topology of the basis of the gauge-natural bundle (see e.g. the review in [19]).

We shall show that, when a gauge-natural invariant variational principle is assigned, to determine canonical (i.e. completely determined by the variational problem and its invariance properties) covariant conservation laws, the vertical part of gauge-natural lifts of infinitesimal principal automorphisms must satisfy generalized Jacobi equations for the gauge-natural invariant Lagrangian. Vice versa all vertical parts of gauge-natural lifts of infinitesimal principal automorphisms which are in the kernel of the generalized Jacobi morphism are generators of canonical covariant currents and superpotentials.

This result is the outcoming of the following facts:

1. the role played by the invariance of a given variational problem of order $s$ on a fibered manifold $\boldsymbol{Y} \rightarrow \boldsymbol{X}$ with respect to the contact structure induced by the affine fiberings $\pi_{s}^{s+1}: J_{s+1} \boldsymbol{Y} \rightarrow J_{s} \boldsymbol{Y}$ and its encoding through the Krupka's finite order variational sequence language. It is fundamental to stress that such an invariance has to reflect too in a precise way on the nature of the conserved Noether currents associated with a given gauge-natural invariant Lagrangian (see Remark 4);

2. the Noether Theorems (both of them) take a quite particular form in the case of gauge-natural Lagrangian field theories (see e.g. [6, 19]) due to the fact that the generalized Lie derivative of sections of the gauge-natural bundles has special linearity properties and it is related with the vertical part (with respect to the splitting induced by the contact structure) of gauge-natural lifts of right-invariant (also called principal) infinitesimal automorphisms of the underlying principal bundle (structure bundle);

3 . the second variation of the action functional can be conveniently represented in the finite order variational sequence framework in terms of iterated variational Lie derivatives (the quotient Lie derivative of variational morphisms for first introduced in [10]) with respect to vertical parts of gauge-natural lifts of principal infinitesimal automorphisms. In particular, by resorting to the Second Noether Theorem, in 8, 9] the second variation has been related with the generalized Jacobi morphism and in [21] the relation of the kernel of generalized gauge-natural Jacobi morphism with the kernel of a fundamental morphism, the (Bergman-)Bianchi morphism, has been explicitly clarified in order to characterize Bianchi identities for geometric field theories in terms of a special class of gauge-natural lifts of infinitesimal principal automorphisms, namely those which have their vertical part in the kernel of the generalized gauge-natural Jacobi morphism. 
Here we claim that the indeterminacy appearing in the derivation of gaugenatural conserved charges (see the interesting papers [13, 19]) - i.e. the difficulty of relating in a natural way infinitesimal gauge transfomations with infinitesimal transformations of the basis manifold (e.g. of space-time) - can be solved by requiring the second variation to be zero too. Historically Jacobi equations were related to the so-called accessory problem (see, e.g. 3] 22]), where they are directly obtained as the variation of the Euler-Lagrange equations of a given Lagrangian. Thus they can be characterized via the Second Noether Theorem.

\section{Variational sequences on gauge-natural bun- dles}

Our framework is a fibered manifold $\pi: \boldsymbol{Y} \rightarrow \boldsymbol{X}$, with $\operatorname{dim} \boldsymbol{X}=n$ and $\operatorname{dim} \boldsymbol{Y}=$ $n+m$. For $s \geq q \geq 0$ integers we are concerned with the $s$-jet space $J_{s} \boldsymbol{Y}$ of $s$-jet prolongations of (local) sections of $\pi$ (see e.g. [15, 18, 23]); in particular, we set $J_{0} \boldsymbol{Y} \equiv \boldsymbol{Y}$. We recall the natural fiberings $\pi_{q}^{s}: J_{s} \boldsymbol{Y} \rightarrow J_{q} \boldsymbol{Y}, s \geq q$, $\pi^{s}: J_{s} \boldsymbol{Y} \rightarrow \boldsymbol{X}$, and, among these, the affine fiberings $\pi_{s-1}^{s}$. We denote with $V \boldsymbol{Y}$ the vector subbundle of the tangent bundle $T \boldsymbol{Y}$ of vectors on $\boldsymbol{Y}$ which are vertical with respect to the fibering $\pi$.

Charts on $\boldsymbol{Y}$ adapted to $\pi$ are denoted by $\left(x^{\sigma}, y^{i}\right)$. Greek indices $\sigma, \mu, \ldots$ run from 1 to $n$ and they label basis coordinates, while Latin indices $i, j, \ldots$ run from 1 to $m$ and label fibre coordinates, unless otherwise specified. We denote multi-indices of dimension $n$ by boldface Greek letters such as $\boldsymbol{\alpha}=\left(\alpha_{1}, \ldots, \alpha_{n}\right)$, with $0 \leq \alpha_{\mu}, \mu=1, \ldots, n$; by an abuse of notation, we denote with $\sigma$ the multi-index such that $\alpha_{\mu}=0$, if $\mu \neq \sigma, \alpha_{\mu}=1$, if $\mu=\sigma$. We also set $|\boldsymbol{\alpha}|:=\alpha_{1}+\cdots+\alpha_{n}$ and $\boldsymbol{\alpha} !:=\alpha_{1} ! \ldots \alpha_{n} !$. The charts induced on $J_{s} \boldsymbol{Y}$ are denoted by $\left(x^{\sigma}, y_{\boldsymbol{\alpha}}^{i}\right)$, with $0 \leq|\boldsymbol{\alpha}| \leq s$; in particular, we set $y_{0}^{i} \equiv y^{i}$. The local vector fields and forms of $J_{s} \boldsymbol{Y}$ induced by the above coordinates are denoted by $\left(\partial_{i}^{\boldsymbol{\alpha}}\right)$ and $\left(d_{\boldsymbol{\alpha}}^{i}\right)$, respectively.

In the theory of variational sequences a fundamental role is played by the contact maps on jet spaces (see [17, 18, 25]). Namely, for $s \geq 1$, we consider the natural complementary fibered morphisms over $J_{s} \boldsymbol{Y} \rightarrow J_{s-1} \boldsymbol{Y}$

$$
\mathcal{D}: J_{s} \boldsymbol{Y} \underset{\boldsymbol{X}}{\times} T \boldsymbol{X} \rightarrow T J_{s-1} \boldsymbol{Y}, \quad \vartheta: J_{s} \boldsymbol{Y} \underset{J_{s-1} \boldsymbol{Y}}{\times} T J_{s-1} \boldsymbol{Y} \rightarrow V J_{s-1} \boldsymbol{Y},
$$

with coordinate expressions, for $0 \leq|\boldsymbol{\alpha}| \leq s-1$, given by

$$
\mathcal{D}=d^{\lambda} \otimes \mathcal{D}_{\lambda}=d^{\lambda} \otimes\left(\partial_{\lambda}+y_{\boldsymbol{\alpha}+\lambda}^{j} \partial_{j}^{\boldsymbol{\alpha}}\right), \vartheta=\vartheta_{\boldsymbol{\alpha}}^{j} \otimes \partial_{j}^{\boldsymbol{\alpha}}=\left(d_{\boldsymbol{\alpha}}^{j}-y_{\boldsymbol{\alpha}+\lambda}^{j} d^{\lambda}\right) \otimes \partial_{j}^{\boldsymbol{\alpha}}
$$

which induce the following natural splitting:

$$
J_{s} \boldsymbol{Y} \underset{J_{s-1} \boldsymbol{Y}}{\times} T^{*} J_{s-1} \boldsymbol{Y}=\left(J_{s} \boldsymbol{Y} \underset{J_{s-1} \boldsymbol{Y}}{\times} T^{*} \boldsymbol{X}\right) \oplus \mathcal{C}_{s-1}^{*}[\boldsymbol{Y}]
$$

where $\mathcal{C}_{s-1}^{*}[\boldsymbol{Y}] \simeq J_{s} \boldsymbol{Y} \underset{J_{s-1} \boldsymbol{Y}}{\times} V^{*} J_{s-1} \boldsymbol{Y}$ 
If $f: J_{s} \boldsymbol{Y} \rightarrow \mathbb{R}$ is a function, then we set $D_{\sigma} f:=\mathcal{D}_{\sigma} f, D_{\boldsymbol{\alpha}+\sigma} f:=D_{\sigma} D_{\boldsymbol{\alpha}} f$, where $D_{\sigma}$ is the standard formal derivative. Given a vector field $\Xi: J_{s} \boldsymbol{Y} \rightarrow$ $T J_{s} \boldsymbol{Y}$, the splitting (11) yields $\Xi \circ \pi_{s}^{s+1}=\Xi_{H}+\Xi_{V}$ where, if $\Xi=\Xi^{\gamma} \partial_{\gamma}+\Xi_{\alpha}^{i} \partial_{i}^{\alpha}$, then we have $\Xi_{H}=\Xi^{\gamma} D_{\gamma}$ and $\Xi_{V}=\left(\Xi_{\boldsymbol{\alpha}}^{i}-y_{\boldsymbol{\alpha}+\gamma}^{i} \Xi^{\gamma}\right) \partial_{i}^{\boldsymbol{\alpha}}$. We shall call $\Xi_{H}$ and $\Xi_{V}$ the horizontal and the vertical part of $\Xi$, respectively.

The splitting (1) induces also a decomposition of the exterior differential on $\boldsymbol{Y},\left(\pi_{s-1}^{s}\right)^{*} \circ d=d_{H}+d_{V}$, where $d_{H}$ and $d_{V}$ are defined to be the horizontal and vertical differential. The action of $d_{H}$ and $d_{V}$ on functions and 1-forms on $J_{s} \boldsymbol{Y}$ uniquely characterizes $d_{H}$ and $d_{V}$ (see, e.g., 23, 25, for more details). A projectable vector field on $\boldsymbol{Y}$ is defined to be a pair $(\Xi, \xi)$, where $\Xi: \boldsymbol{Y} \rightarrow T \boldsymbol{Y}$ and $\xi: \boldsymbol{X} \rightarrow T \boldsymbol{X}$ are vector fields and $\Xi$ is a fibered morphism over $\xi$. If there is no danger of confusion, we will denote simply by $\Xi$ a projectable vector field $(\Xi, \xi)$. A projectable vector field $(\Xi, \xi)$ can be prolonged by the flow functor to a projectable vector field $\left(j_{s} \Xi, \xi\right)$, the coordinate expression of which can be found e.g. in 17, 18, 23, 25]; in particular, we have the following expressions $\left(j_{s} \Xi\right)_{H}=\xi^{\sigma} D_{\sigma},\left(j_{s} \Xi\right)_{V}=D_{\boldsymbol{\alpha}}\left(\Xi_{V}\right)^{i} \partial_{i}^{\alpha}$, with $\left(\Xi_{V}\right)^{i}=\xi^{i}-y_{\sigma}^{i} \xi^{\sigma}$, for the horizontal and the vertical part of $j_{s} \Xi$, respectively. From now on, by an abuse of notation, we will write simply $j_{s} \Xi_{H}$ and $j_{s} \Xi_{V}$. In particular, we stress that $j_{s} \Xi_{V}$ can be seen as a fibered morphism: $j_{s} \Xi_{V}: J_{s+1} \boldsymbol{Y} \underset{J_{s} \boldsymbol{Y}}{\times} J_{s} \boldsymbol{Y} \rightarrow J_{s+1} \boldsymbol{Y} \underset{J_{s} \boldsymbol{Y}}{\times} J_{s} V \boldsymbol{Y}$.

\subsection{Gauge-natural bundles}

In the following, we shall develop a suitable geometrical setting which enables us to define and investigate the fundamental concept of conserved quantity in gauge-natural Lagrangian field theories.

An important generalization of natural field theories 24] to gauge fields theories passed through the concept of jet prolongation of a principal bundle and the introduction of a very important geometric construction, namely the gauge-natural bundle functor [5, 15].

Let $\boldsymbol{P} \rightarrow \boldsymbol{X}$ be a principal bundle with structure group $\boldsymbol{G}$. Let $r \leq k$ be integers and $\boldsymbol{W}^{(r, k)} \boldsymbol{P}:=J_{r} \boldsymbol{P} \underset{\boldsymbol{X}}{\times} L_{k}(\boldsymbol{X})$, where $L_{k}(\boldsymbol{X})$ is the bundle of $k$-frames in $\boldsymbol{X}$ 5, 15, $\boldsymbol{W}^{(r, k)} \boldsymbol{G}:=J_{r} \boldsymbol{G} \odot G L_{k}(n)$ the semidirect product with respect to the action of $G L_{k}(n)$ on $J_{r} \boldsymbol{G}$ given by the jet composition and $G L_{k}(n)$ is the group of $k$-frames in $\mathbb{R}^{n}$. Here we denote by $J_{r} \boldsymbol{G}$ the space of $(r, n)$-velocities on $\boldsymbol{G}$. The bundle $\boldsymbol{W}^{(r, k)} \boldsymbol{P}$ is a principal bundle over $\boldsymbol{X}$ with structure group $\boldsymbol{W}^{(r, k)} \boldsymbol{G}$. The right action of $\boldsymbol{W}^{(r, k)} \boldsymbol{G}$ on the fibers of $\boldsymbol{W}^{(r, k)} \boldsymbol{P}$ is defined by the composition of jets (see, e.g., [15]).

Definition 1 The principal bundle $\boldsymbol{W}^{(r, k)} \boldsymbol{P}$ (resp. the Lie group $\boldsymbol{W}^{(r, k)} \boldsymbol{G}$ ) is said to be the gauge-natural prolongation of order $(r, k)$ of $\boldsymbol{P}$ (resp. of $\boldsymbol{G}$ ).

Definition 2 We define the vector bundle over $\boldsymbol{X}$ of right-invariant infinitesimal automorphisms of $\boldsymbol{P}$ by setting $\mathcal{A}=T \boldsymbol{P} / \boldsymbol{G}$.

For $r \leq k$ we also define the vector bundle over $\boldsymbol{X}$ of right invariant infinitesimal automorphisms of $\boldsymbol{W}^{(r, k)} \boldsymbol{P}$ by setting $\mathcal{A}^{(r, k)}:=T \boldsymbol{W}^{(r, k)} \boldsymbol{P} / \boldsymbol{W}^{(r, k)} \boldsymbol{G}$. 
Let $\boldsymbol{F}$ be any manifold and $\zeta: \boldsymbol{W}^{(r, k)} \boldsymbol{G} \times \boldsymbol{F} \rightarrow \boldsymbol{F}$ be a left action of $\boldsymbol{W}^{(r, k)} \boldsymbol{G}$ on $\boldsymbol{F}$. There is a naturally defined right action of $\boldsymbol{W}^{(r, k)} \boldsymbol{G}$ on $\boldsymbol{W}^{(r, k)} \boldsymbol{P} \times \boldsymbol{F}$ so that we can associate in a standard way to $\boldsymbol{W}^{(r, k)} \boldsymbol{P}$ the bundle, on the given basis $\boldsymbol{X}, \boldsymbol{Y}_{\zeta}:=\boldsymbol{W}^{(r, k)} \boldsymbol{P} \times_{\zeta} \boldsymbol{F}[5,15]$.

Definition 3 We say $\left(\boldsymbol{Y}_{\zeta}, \boldsymbol{X}, \pi_{\zeta} ; \boldsymbol{F}, \boldsymbol{G}\right)$ to be the gauge-natural bundle of order $(r, k)$ associated to the principal bundle $\boldsymbol{W}^{(r, k)} \boldsymbol{P}$ by means of the left action $\zeta$ of the group $\boldsymbol{W}^{(r, k)} \boldsymbol{G}$ on the manifold $\boldsymbol{F}$.

Remark 1 A principal automorphism $\Phi$ of $\boldsymbol{W}^{(r, k)} \boldsymbol{P}$ induces an automorphism of the gauge-natural bundle by:

$$
\Phi_{\zeta}: \boldsymbol{Y}_{\zeta} \rightarrow \boldsymbol{Y}_{\zeta}:\left[\left(j_{r}^{x} \gamma, j_{k}^{0} t\right), \hat{f}\right]_{\zeta} \mapsto\left[\Phi\left(j_{r}^{x} \gamma, j_{k}^{0} t\right), \hat{f}\right]_{\zeta}
$$

where $\hat{f} \in \boldsymbol{F}$ and $[\cdot, \cdot]_{\zeta}$ is the equivalence class induced by the action $\zeta$.

Denote by $\mathcal{T}_{\boldsymbol{X}}$ and $\mathcal{A}^{(r, k)}$ the sheaf of vector fields on $\boldsymbol{X}$ and the sheaf of right invariant vector fields on $\boldsymbol{W}^{(r, k)} \boldsymbol{P}$, respectively. A functorial mapping $\mathfrak{G}$ is defined which lifts any right-invariant local automorphism $(\Phi, \phi)$ of the principal bundle $W^{(r, k)} \boldsymbol{P}$ into a unique local automorphism $\left(\Phi_{\zeta}, \phi\right)$ of the associated bundle $\boldsymbol{Y}_{\zeta}$. Its infinitesimal version associates to any $\underline{\Xi} \in \mathcal{A}^{(r, k)}$, projectable over $\xi \in \mathcal{T}_{\boldsymbol{X}}$, a unique projectable vector field $\hat{\Xi}:=\mathfrak{G}(\bar{\Xi})$ on $\boldsymbol{Y}_{\zeta}$ in the following way:

$$
\mathfrak{G}: \boldsymbol{Y}_{\zeta} \underset{\boldsymbol{X}}{\times} \mathcal{A}^{(r, k)} \rightarrow T \boldsymbol{Y}_{\zeta}:(\boldsymbol{y}, \bar{\Xi}) \mapsto \hat{\Xi}(\boldsymbol{y})
$$

where, for any $\boldsymbol{y} \in \boldsymbol{Y}_{\zeta}$, one sets: $\hat{\Xi}(\boldsymbol{y})=\frac{d}{d t}\left[\left(\Phi_{\zeta t}\right)(\boldsymbol{y})\right]_{t=0}$, and $\Phi_{\zeta t}$ denotes the (local) flow corresponding to the gauge-natural lift of $\Phi_{t}$.

This mapping fulfils the following properties:

1. $\mathfrak{G}$ is linear over $i d_{\boldsymbol{Y}_{\zeta}}$;

2. we have $T \pi_{\zeta} \circ \mathfrak{G}=i d_{T \boldsymbol{X}} \circ \bar{\pi}^{(r, k)}$, where $\bar{\pi}^{(r, k)}$ is the natural projection $\boldsymbol{Y}_{\zeta} \underset{\boldsymbol{X}}{\times} \mathcal{A}^{(r, k)} \rightarrow T \boldsymbol{X}$

3. $\mathfrak{G}$ is a homomorphism of Lie algebras: for any pair $(\bar{\Lambda}, \bar{\Xi})$ of vector fields in $\mathcal{A}^{(r, k)}$, we have $\mathfrak{G}([\bar{\Lambda}, \bar{\Xi}])=[\mathfrak{G}(\bar{\Lambda}), \mathfrak{G}(\bar{\Xi})]$;

4. in coordinates $\mathfrak{G}=d^{\mu} \otimes \partial_{\mu}+d_{\nu}^{A} \otimes\left(\mathcal{Z}_{A}^{i \nu} \partial_{i}\right)+d_{\boldsymbol{\lambda}}^{\nu} \otimes\left(\mathcal{Z}_{\nu}^{i \boldsymbol{\lambda}} \partial_{i}\right)$, with $0<|\boldsymbol{\nu}|<k$, $1<|\boldsymbol{\lambda}|<r$ and $\mathcal{Z}_{A}^{i \nu}, \mathcal{Z}_{\nu}^{i \boldsymbol{\lambda}} \in C^{\infty}\left(\boldsymbol{Y}_{\zeta}\right)$ are suitable functions which depend on the bundle, precisely on the fibers (see [15]).

Definition 4 The map $\mathfrak{G}$ is called the gauge-natural lifting functor. The projectable vector field $(\hat{\Xi}, \xi) \equiv \mathfrak{G}((\bar{\Xi}, \xi))$ is called the gauge-natural lift of $(\bar{\Xi}, \xi)$ to the bundle $\boldsymbol{Y}_{\zeta}$. 
We shall consider variation vector fields which are vertical parts of gaugenatural lifts of infinitesimal principal automorphisms. We recall that, due to the very definition of generalized Lie derivative of sections of gauge-natural bundles, variation vector fields are in fact formal Lie derivatives of sections with respect to gauge-natural lifts (see the item 4 in the following). This will enables us to realize morphisms such as the Jacobi or the Bianchi morphisms in a very suitable way for our purposes.

Definition 5 (Lie derivative of sections.) Let $\gamma$ be a (local) section of the gauge-natural bundle $\boldsymbol{Y}_{\zeta}, \bar{\Xi} \in \mathcal{A}^{(r, k)}$ and $\hat{\Xi}$ its gauge-natural lift. Following [15] we define the generalized Lie derivative of $\gamma$ along the vector field $\hat{\Xi}$ to be the

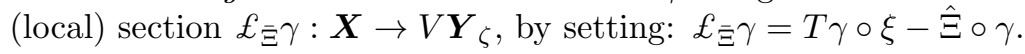

Remark 2 This section is a vertical prolongation of $\gamma$, i.e. it satisfies the property: $\nu_{\boldsymbol{Y}_{\zeta}} \circ £_{\Xi \gamma}=\gamma$, where $\nu_{\boldsymbol{Y}_{\zeta}}$ is the projection $\nu_{\boldsymbol{Y}_{\zeta}}: V \boldsymbol{Y}_{\zeta} \rightarrow \boldsymbol{Y}_{\zeta}$. Its coordinate expression is given by $\left(£_{\bar{\Xi}} \gamma\right)^{i}=\xi^{\sigma} \partial_{\sigma} \gamma^{i}-\hat{\Xi}^{i}(\gamma)$. As customary we denote it by $£_{\bar{\Xi}} \gamma$ and not by $£_{\hat{\Xi}} \gamma$ because of the functorial correspondence between $\bar{\Xi}$ and $\hat{\Xi}$.

Remark 3 The Lie derivative operator acting on sections of gauge-natural bundles satisfies the following properties:

1. for any vector field $\bar{\Xi} \in \mathcal{A}^{(r, k)}$, the mapping $\gamma \mapsto £ \equiv \gamma$ is a first-order quasilinear differential operator;

2. for any local section $\gamma$ of $\boldsymbol{Y}_{\zeta}$, the mapping $\bar{\Xi} \mapsto £ \equiv \gamma$ is a linear differential operator;

3. we can regard $£_{\Xi}: J_{1} \boldsymbol{Y}_{\zeta} \rightarrow V \boldsymbol{Y}_{\zeta}$ as a morphism over the basis $\boldsymbol{X}$. In this case it is meaningful to consider the (standard) jet prolongation of $£_{\bar{\Xi}}$, denoted by $j_{s} £_{\Xi}: J_{s+1} \boldsymbol{Y}_{\zeta} \rightarrow V J_{s} \boldsymbol{Y}_{\zeta}$. By using the canonical isomorphism $V J_{s} \boldsymbol{Y}_{\zeta} \simeq J_{s} V \boldsymbol{Y}_{\zeta}$, we have $£_{\Xi}\left(j_{s} \gamma\right)=j_{s}\left(£_{\Xi} \gamma\right)$, for any (local) section $\gamma$ of $\boldsymbol{Y}_{\zeta}$ and for any (local) vector field $\bar{\Xi} \in \mathcal{A}^{(r, k)}$.

4. as a consequence of linearity properties of gauge-natural lifts, we have $j_{s} \hat{\Xi}_{V}(\gamma)=-£_{j_{s} \Xi} \gamma$. In particular, we can consider the Lie derivative of sections, $£$, as a bundle morphism [21]:

$$
£: J_{s+1}\left(\boldsymbol{Y}_{\zeta} \underset{\boldsymbol{X}}{\times} \mathcal{A}^{(r, k)}\right) \rightarrow J_{s+1} \boldsymbol{Y}_{\zeta} \underset{J_{s} \boldsymbol{Y}_{\zeta}}{\times} V J_{s} \boldsymbol{Y}_{\zeta}
$$

\subsection{Variational Lie derivative of variational morphisms}

For the sake of simplifying notation, sometimes, we will omit the subscript $\zeta$, so that all our considerations shall refer to $\boldsymbol{Y}$ as a gauge-natural bundle as defined above. 
We shall be here concerned with some distinguished sheaves of forms on jet spaces [17, 23, 25]. We shall in particular follow notation given in [25] to which the reader is referred for details. For $s \geq 0$, we consider the standard sheaves $\Lambda_{s}^{p}$ of $p$-forms on $J_{s} \boldsymbol{Y}$. For $0 \leq q \leq s$, we consider the sheaves $\mathcal{H}_{(s, q)}^{p}$ and $\mathcal{H}_{s}^{p}$ of horizontal forms with respect to the projections $\pi_{q}^{s}$ and $\pi_{0}^{s}$, respectively. For $0 \leq q<s$, we consider the subsheaves $\mathcal{C}_{(s, q)}^{p} \subset \mathcal{H}_{(s, q)}^{p}$ and $\mathcal{C}^{p}{ }_{s} \subset \mathcal{C}_{(s+1, s)}^{p}$ of contact forms, i.e. horizontal forms valued into $\mathcal{C}_{s}^{*}[\boldsymbol{Y}]$ (they have the property of vanishing along any section of the gauge-natural bundle).

According to [17, 25, the fibered splitting (11) yields the sheaf splitting $\mathcal{H}_{(s+1, s)}^{p}=\bigoplus_{t=0}^{p} \mathcal{C}_{(s+1, s)}^{p-t} \wedge \mathcal{H}_{s+1}^{t}$, which restricts to the inclusion $\Lambda_{s}^{p} \subset \bigoplus_{t=0}^{p}$ $\mathcal{C}^{p-t}{ }_{s} \wedge \mathcal{H}^{t, h}{ }_{s+1}$, where $\mathcal{H}^{p, h}{ }_{s+1}:=h\left(\Lambda_{s}^{p}\right)$ for $0<p \leq n$ and the surjective map $h$ is defined to be the restriction to $\Lambda_{s}^{p}$ of the projection of the above splitting onto the non-trivial summand with the highest value of $t$. By an abuse of notation, let us denote by $d \operatorname{ker} h$ the sheaf generated by the presheaf $d \operatorname{ker} h$ in the standard way. We set $\Theta_{s}^{*}:=\operatorname{ker} h+d \operatorname{ker} h$.

The $s$-th order quotient variational sequence associated with the fibered manifold $\boldsymbol{Y} \rightarrow \boldsymbol{X}$ :

$0 \rightarrow \mathbb{R}_{Y} \longrightarrow \Lambda_{s}^{0} \stackrel{\mathcal{E}_{0}}{\longrightarrow} \Lambda_{s}^{1} / \Theta_{s}^{1} \stackrel{\mathcal{E}_{1}}{\longrightarrow} \Lambda_{s}^{2} / \Theta_{s}^{2} \stackrel{\mathcal{E}_{2}}{\longrightarrow} \ldots \stackrel{\mathcal{E}_{I-1}}{\longrightarrow} \Lambda_{s}^{I} / \Theta_{s}^{I} \stackrel{\mathcal{E}_{I}}{\longrightarrow} \Lambda_{s}^{I+1} \stackrel{d}{\longrightarrow} 0$,

has been introduced by Krupka 17.

To the aim of characterizing some fundamental morphisms for the calculus of variations as sections of quotient sheaves and as corresponding differential quotient morphisms, let us consider the truncated variational sequence:

$$
0 \rightarrow \mathbb{R}_{Y} \rightarrow \mathcal{V}_{s}^{0} \stackrel{\mathcal{E}_{0}}{\longrightarrow} \mathcal{V}_{s}^{1} \stackrel{\mathcal{E}_{1}}{\longrightarrow} \ldots \stackrel{\mathcal{E}_{n}}{\longrightarrow} \mathcal{V}_{s}^{n+1} \stackrel{\mathcal{E}_{n+1}}{\longrightarrow} \mathcal{E}_{n+1}\left(\mathcal{V}_{s}^{n+1}\right) \stackrel{\mathcal{E}_{n+2}}{\longrightarrow} 0
$$

where, following [25], the sheaves $\mathcal{V}_{s}^{p}:=\mathcal{C}_{s}^{p-n} \wedge \mathcal{H}^{n, h}{ }_{s+1} / h(d$ ker $h)$ with $0 \leq p \leq$ $n+2$ are suitable representations of the corresponding quotient sheaves in the variational sequence by means of sheaves of sections of tensor bundles.

Let $\alpha \in \mathcal{C}_{s}^{1} \wedge \mathcal{H}_{s+1}^{n, h} \subset \mathcal{V}_{s+1}^{n+1}$. Then there is a unique pair of sheaf morphisms (14, 16, 25)

$$
E_{\alpha} \in \mathcal{C}_{(2 s, 0)}^{1} \wedge \mathcal{H}^{n, h}{ }_{2 s+1}, \quad F_{\alpha} \in \mathcal{C}_{(2 s, s)}^{1} \wedge \mathcal{H}^{n, h}{ }_{2 s+1},
$$

such that $\left(\pi_{s+1}^{2 s+1}\right)^{*} \alpha=E_{\alpha}-F_{\alpha}$, and $F_{\alpha}$ is locally of the form $F_{\alpha}=d_{H} p_{\alpha}$, with $p_{\alpha} \in \mathcal{C}_{(2 s-1, s-1)}^{1} \wedge \mathcal{H}^{n-1}{ }_{2 s}$.

Definition 6 Let $\gamma \in \Lambda_{s}^{n+1}$. The morphism $E_{h(\gamma)} \in \mathcal{V}_{s}^{n+1}$ is called the generalized Euler-Lagrange morphism associated with $\gamma$.

Let $\eta \in \mathcal{C}_{s}^{1} \wedge \mathcal{C}_{(s, 0)}^{1} \wedge \mathcal{H}^{n, h}{ }_{s+1} \subset \mathcal{V}_{s+1}^{n+2}$, then there is a unique morphism

$$
K_{\eta} \in \mathcal{C}_{(2 s, s)}^{1} \otimes \mathcal{C}_{(2 s, 0)}^{1} \wedge \mathcal{H}^{n, h}{ }_{2 s+1},
$$


such that, for all $\Xi: \boldsymbol{Y} \rightarrow V \boldsymbol{Y}, E_{\left.j_{s} \Xi\right\rfloor \eta}=C_{1}^{1}\left(j_{2 s} \Xi \otimes K_{\eta}\right)$, where $C_{1}^{1}$ stands for tensor contraction on the first factor and $\rfloor$ denotes inner product (see [16, 25]). Furthermore, there is a unique pair of sheaf morphisms

$$
H_{\eta} \in \mathcal{C}_{(2 s, s)}^{1} \wedge \mathcal{C}_{(2 s, 0)}^{1} \wedge \mathcal{H}_{2 s+1}^{n, h}, \quad G_{\eta} \in \mathcal{C}_{(2 s, s)}^{2} \wedge \mathcal{H}_{2 s+1}^{n, h}
$$

such that $\left(\pi_{s+1}^{2 s+1}\right)^{*} \eta=H_{\eta}-G_{\eta}$ and $H_{\eta}=\frac{1}{2} A\left(K_{\eta}\right)$, where $A$ stands for antisymmetrisation. Moreover, $G_{\eta}$ is locally of the type $G_{\eta}=d_{H} q_{\eta}$, where $q_{\eta} \in \mathcal{C}_{(2 s-1, s-1)}^{2} \wedge \mathcal{H}^{n-1}{ }_{2 s}$, hence $[\eta]=\left[H_{\eta}\right][16,25]$.

Definition 7 Let $\gamma \in \Lambda_{s}^{n+1}$. The morphism $H_{h d \gamma} \equiv H_{\left[\mathcal{E}_{n+1}(\gamma)\right]}$, where square brackets denote equivalence class, is called the generalized Helmholtz morphism.

The standard Lie derivative of fibered morphisms with respect to a projectable vector field $j_{s} \Xi$ passes to the quotient in the variational sequence, thus defining a new quotient operator (introduced in [10]), the variational Lie derivative $\mathcal{L}_{j_{s} \Xi}$, acting on equivalence classes of fibered morphisms which are sections of the quotient sheaves in the variational sequence. Thus variational Lie derivatives of generalized Lagrangians or Euler-Lagrange morphisms can be conveniently represented as equivalence classes in $\mathcal{V}_{s}^{n}$ and $\mathcal{V}_{s}^{n+1}$. In particular, the following two results hold true [10].

Theorem 1 Let $[\alpha]=h(\alpha) \in \mathcal{V}_{s}^{n}$. Then we have locally (up to pull-backs)

$$
\left.\left.\left.\mathcal{L}_{j_{s} \Xi}(h(\alpha))=\Xi_{V}\right\rfloor \mathcal{E}_{n}(h(\alpha))+d_{H}\left(j_{2 s} \Xi_{V}\right\rfloor p_{d_{V} h(\alpha)}+\xi\right\rfloor h(\alpha)\right) .
$$

Theorem 2 Let $\alpha \in \Lambda_{s}^{n+1}$. Then we have globally (up to pull-backs)

$$
\left.\mathcal{L}_{j_{s}} \Xi[\alpha]=\mathcal{E}_{n}\left(j_{s+1} \Xi_{V}\right\rfloor h(\alpha)\right)+C_{1}^{1}\left(j_{s} \Xi_{V} \otimes K_{h d \alpha}\right) .
$$

\subsection{Generalized gauge-natural Jacobi morphisms}

We recall some previous results concerning the representation of generalized gauge-natural Jacobi morphisms in variational sequences and their relation with the second variation of a generalized gauge-natural invariant Lagrangian [21.

Definition 8 Let $\alpha: J_{s} \boldsymbol{Y} \rightarrow \stackrel{p}{\wedge} T^{*} J_{s} \boldsymbol{Y}$. Let $\psi_{t_{k}}^{k}$, with $1 \leq k \leq i$, be the flows generated by an $i$-tuple $\left(\Xi_{1}, \ldots, \Xi_{i}\right)$ of (vertical, although actually it is enough that they are projectable) vector fields on $\boldsymbol{Y}$ and let $\Gamma_{i}$ be the $i$-th formal variation generated by the $\Xi_{k}$ 's (to which we shall refer as variation vector fields) and defined, for each $\boldsymbol{y} \in \boldsymbol{Y}$, by $\Gamma_{i}\left(t_{1}, \ldots, t_{i}\right)(\boldsymbol{y})=\psi_{t_{i}}^{i} \circ \ldots \circ \psi_{t_{1}}^{1}(\boldsymbol{y})$. We define the $i$-th formal variation of the morphism $\alpha$ to be

$$
\delta^{i} \alpha:=\left.\frac{\partial^{i}}{\partial t_{1} \ldots \partial t_{i}}\right|_{t_{1}, \ldots, t_{i}=0}\left(\alpha \circ j_{s} \Gamma_{i}\left(t_{1}, \ldots, t_{i}\right)(\boldsymbol{y})\right) .
$$


The following Lemma states the relation between the $i$-th formal variation of a morphism and its iterated Lie derivative [1], 21.

Lemma 1 Let $\alpha: J_{s} \boldsymbol{Y} \rightarrow \stackrel{p}{\wedge} T^{*} J_{s} \boldsymbol{Y}$ and $L_{j_{s} \Xi_{k}}$ be the Lie derivative operator acting on differential fibered morphism.

Let $\Gamma_{i}$ be the $i$-th formal variation generated by variation vector fields $\Xi_{k}$, $1 \leq k \leq i$ on $\boldsymbol{Y}$. Then we have

$$
\delta^{i} \alpha=L_{j_{s} \Xi_{1}} \ldots L_{j_{s} \Xi_{i}} \alpha .
$$

Let $\alpha \in\left(\mathcal{V}_{s}^{n}\right)_{\boldsymbol{Y}}$. The operator $\delta^{i}$ passes to the quotient in the variational sequence. We shall call the quotient operator the $i$-th variational vertical derivative. We have $\delta^{i}[\alpha]:=\left[\delta^{i} \alpha\right]=\left[L_{\Xi_{i}} \ldots L_{\Xi_{1}} \alpha\right]=\mathcal{L}_{\Xi_{i}} \ldots \mathcal{L}_{\Xi_{1}}[\alpha]$.

Let now variation vector fields be vertical parts of gauge-natural lifts. By resorting to the Second Noether Theorem, we have the following characterization of the second variational vertical derivative of a generalized Lagrangian 21 which in fact enable us to relate the second variation with the morphism $K_{\eta}$ defined by Eq. (5) (for $\eta=h d \delta \lambda$ ).

First of all we fix some preliminary properties of gauge-natural lifts.

Lemma 2 Let $j_{s} \hat{\Xi}$ be the s-jet prolongation of $\hat{\Xi}$ which is a vector field on $J_{s} \boldsymbol{Y}_{\zeta}$. It turns out then that $j_{s} \mathfrak{G}(\bar{\Xi})=\mathfrak{G}\left(j_{s} \overline{\bar{\Xi}}\right)$.

PROOF. Owing to linearity properties of the Lie derivative of sections of gauge-natural bundles and since $j_{s} \hat{\Xi}_{V}=-£_{j_{s}} \bar{\Xi}$, the statement is a consequence of Proposition 15.5 in [15].

QED

Let $j_{s} \hat{\Xi}_{V}$ be the vertical part according to the splitting (1). We shall denote by $j_{s} \bar{\Xi}_{V}$ the induced section of the vector bundle $\mathcal{A}^{(r+s, k+s)}$ following the Lemma above. The set of all sections of this kind defines a vector subbundle of $J_{s} \mathcal{A}^{(r, k)}$ which, by an abuse of notation, we shall denote by $V J_{s} \mathcal{A}^{(r, k)}$. Let $j_{s} \hat{\Xi}_{V}$ be variation vector fields and let $\delta_{\mathfrak{G}}^{2} \lambda$ be the variation of $\lambda$ with respect to such variation vector fields.

Proposition 1 Let $\lambda \in\left(\mathcal{V}_{s}^{n}\right)_{\boldsymbol{Y}}$ and let $\mathfrak{G}(\bar{\Xi})_{V}$ be a variation vector field. Let

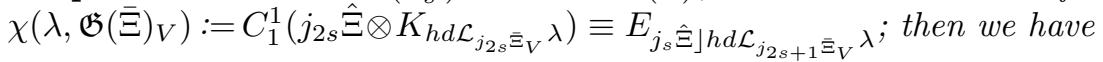

$$
\left.\delta_{\mathfrak{G}}^{2} \lambda=\left[\mathcal{E}_{n}\left(j_{2 s} \Xi\right\rfloor h \delta \lambda\right)+\mathcal{J}\left(\lambda, \mathfrak{G}(\bar{\Xi})_{V}\right)\right]
$$

where [] denotes the equivalence class in the variational sequence, while $\mathcal{J}\left(\lambda, \mathfrak{G}(\bar{\Xi})_{V}\right):=E_{\chi\left(\lambda, \mathfrak{G}(\bar{\Xi})_{V}\right)}$.

Definition 9 Let $\bar{\Xi} \in \mathcal{A}^{(r, k)}$. We call the morphism $\mathcal{J}\left(\lambda, \mathfrak{G}(\bar{\Xi})_{V}\right)$ the gaugenatural generalized Jacobi morphism associated with the Lagrangian $\lambda$ and the gauge-natural lift $\mathfrak{G}(\bar{\Xi})_{V}$.

The morphism $\mathcal{J}\left(\lambda, \mathfrak{G}(\bar{\Xi})_{V}\right)$ is a linear morphism with respect to the projection $J_{4 s} \boldsymbol{Y}_{\zeta} \underset{\boldsymbol{X}}{\times} V J_{4 s} \mathcal{A}^{(r, k)} \rightarrow J_{4 s} \boldsymbol{Y}_{\zeta}$. Notice that, seen as a section of $\left(\mathcal{V}_{s}^{n}\right)_{\boldsymbol{Y} \times V \boldsymbol{X}}$, 
the equivalence class $\left.\left[\mathcal{E}_{n}\left(j_{s} \bar{\Xi}_{V}\right\rfloor h(\delta \lambda)\right)\right]$ vanishes being a local divergence of higher contact forms. This can also be compared with analogous results in [11]. Thus, as a consequence Theorem 2 and Proposition 1$]$ we have the following.

Proposition 2 Let $\delta_{\mathfrak{G}}^{2} \lambda$ be the variation of $\lambda$ with respect to vertical parts of gauge-natural lifts of infinitesimal principal automorphisms. We have:

$$
\left.\left.\left.\mathfrak{G}(\bar{\Xi})_{V}\right\rfloor \mathcal{E}_{n}\left(\mathfrak{G}(\bar{\Xi})_{V}\right\rfloor \mathcal{E}_{n}(\lambda)\right)=\delta_{\mathfrak{G}}^{2} \lambda=\mathcal{E}_{n}\left(\mathfrak{G}(\bar{\Xi})_{V}\right\rfloor h(d \delta \lambda)\right) .
$$

\section{Canonical covariant conserved currents}

In the following we assume that the field equations are generated by means of a variational principle from a Lagrangian which is gauge-natural invariant, i.e. invariant with respect to any gauge-natural lift of infinitesimal right invariant vector fields.

Definition 10 Let $(\hat{\Xi}, \xi)$ be a projectable vector field on $\boldsymbol{Y}_{\zeta}$. Let $\lambda \in \mathcal{V}_{s}^{n}$ be a generalized Lagrangian. We say $\hat{\Xi}$ to be a symmetry of $\lambda$ if $\mathcal{L}_{j_{s+1} \hat{\Xi}} \lambda=0$.

We say $\lambda$ to be a gauge-natural invariant Lagrangian if the gauge-natural lift $(\hat{\Xi}, \xi)$ of any vector field $\bar{\Xi} \in \mathcal{A}^{(r, k)}$ is a symmetry for $\lambda$, i.e. if $\mathcal{L}_{j_{s+1}} \bar{\Xi} \lambda=0$. In this case the projectable vector field $\hat{\Xi} \equiv \mathfrak{G}(\bar{\Xi})$ is called a gauge-natural symmetry of $\lambda$.

Remark 4 As well known, the Second Noether Theorem deals with invariance properties of the Euler-Lagrange equations (so-called generalized symmetries or also Bessel-Hagen symmetries, see e.g. the fundamental papers 24]). Although symmetries of a Lagrangian turn out to be also symmetries of the Euler-Lagrange morphism the converse is not true, in general.

In particular, although for a gauge-natural invariant Lagrangian $\lambda$ we always have $\mathcal{L}_{j_{s}} \bar{\Xi} \lambda=0, \mathcal{L}_{j_{s} \bar{\Xi}_{V}} \lambda$ does not need to be zero in principle; however when the second variation $\delta_{\mathfrak{G}}^{2} \lambda$ is required to vanish then $\mathcal{L}_{j_{s} \Xi_{V}} \mathcal{E}_{n}(\lambda)$ surely vanishes, i.e. $j_{s} \bar{\Xi}_{V}$ is a generalized or Bessel-Hagen symmetry. The symmetries of the Euler-Lagrange morphism (Second Noether Theorem) impose some constraints on the conserved quantities associated with gauge-natural symmetries of $\lambda$ (see e.g. [1].

The First Noether Theorem takes a particularly interesting form in the case of gauge-natural Lagrangians as shown in the following.

Proposition 3 Let $\lambda \in \mathcal{V}_{s}^{n}$ be a gauge-natural Lagrangian and $(\hat{\Xi}, \xi)$ a gaugenatural symmetry of $\lambda$. Then we have $\left.0=-£_{\Xi}\right\rfloor \mathcal{E}_{n}(\lambda)+d_{H}\left(-j_{s} £_{\Xi}\right\rfloor p_{d_{V} \lambda}+$ $\xi\rfloor \lambda)$. Suppose that $\left.\left(j_{2 s+1} \sigma\right)^{*}\left(-£_{\Xi}\right\rfloor \mathcal{E}_{n}(\lambda)\right)=0$. Then, the $(n-1)$-form $\epsilon=$ $\left.\left.-j_{s} £_{\bar{\Xi}}\right\rfloor p_{d_{V} \lambda}+\xi\right\rfloor \lambda$ fulfills the equation $d\left(\left(j_{2 s} \sigma\right)^{*}(\epsilon)\right)=0$.

If $\sigma$ is a critical section for $\mathcal{E}_{n}(\lambda)$, i.e. $\left(j_{2 s+1} \sigma\right)^{*} \mathcal{E}_{n}(\lambda)=0$, the above equation admits a physical interpretation as a so-called weak conservation law for the density associated with $\epsilon$. 
Definition 11 Let $\lambda \in \mathcal{V}_{s}^{n}$ be a gauge-natural Lagrangian and $\bar{\Xi} \in \mathcal{A}^{(r, k)}$. Then the sheaf morphism $\epsilon: J_{2 s} \boldsymbol{Y}_{\zeta} \underset{\boldsymbol{X}}{\times} V J_{2 s} \mathcal{A}^{(r, k)} \rightarrow \mathcal{C}_{2 s}^{*}\left[\mathcal{A}^{(r, k)}\right] \otimes \mathcal{C}_{0}^{*}\left[\mathcal{A}^{(r, k)}\right] \wedge\left({ }^{n-1} T^{*} \boldsymbol{X}\right)$ is said to be a gauge-natural weakly conserved current.

Remark 5 In general, this conserved current is not uniquely defined. In fact, it depends on the choice of $p_{d_{V} \lambda}$, which is not unique, in general (see [25] and references quoted therein).

In gauge-natural Lagrangian theories it is a well known procedure to perform suitable integrations by parts to decompose the conserved current $\epsilon$ into the sum of a conserved current vanishing along solutions of the Euler-Lagrange equations, the so-called reduced current, and the formal divergence of a skewsymmetric (tensor) density called a superpotential (which is defined modulo a divergence). Within such a procedure, the generalized Bianchi identities are in fact necessary and (locally) sufficient conditions for the conserved current $\epsilon$ to be not only closed but also the divergence of a skew-symmetric (tensor) density along solutions of the Euler-Lagrange equations.

The following Lemma is a geometric version of the integration by parts procedure quoted above and it is based on a global decomposition formula of vertical morphisms due to Kolář [14.

Lemma $3 \operatorname{Let} \omega\left(\lambda, \mathfrak{G}(\bar{\Xi})_{V}\right):=£_{\Xi} \mid \mathcal{E}_{n}(\lambda): J_{2 s} \boldsymbol{Y}_{\zeta} \underset{\boldsymbol{X}}{\times} V J_{2 s} \mathcal{A}^{(r, k)} \rightarrow \mathcal{C}_{2 s}^{*}\left[\mathcal{A}^{(r, k)}\right] \otimes$ $\mathcal{C}_{2 s}^{*}\left[\mathcal{A}^{(r, k)}\right] \otimes \mathcal{C}_{0}^{*}\left[\mathcal{A}^{(r, k)}\right] \wedge\left(\wedge T^{*} \boldsymbol{X}\right)$. Then we have globally

$$
\left(\pi_{s+1}^{4 s+1}\right)^{*} \omega\left(\lambda, \mathfrak{G}(\bar{\Xi})_{V}\right)=\beta\left(\lambda, \mathfrak{G}(\bar{\Xi})_{V}\right)+F_{\omega\left(\lambda, \mathfrak{G}(\bar{\Xi})_{V}\right)},
$$

where $\beta\left(\lambda, \mathfrak{G}(\bar{\Xi})_{V}\right) \equiv E_{\omega\left(\lambda, \mathfrak{G}(\bar{\Xi})_{V}\right)}$ :

$: J_{4 s} \boldsymbol{Y}_{\zeta} \underset{\boldsymbol{X}}{\times} V J_{4 s} \mathcal{A}^{(r, k)} \rightarrow \mathcal{C}_{2 s}^{*}\left[\mathcal{A}^{(r, k)}\right] \otimes \mathcal{C}_{0}^{*}\left[\mathcal{A}^{(r, k)}\right] \otimes \mathcal{C}_{0}^{*}\left[\mathcal{A}^{(r, k)}\right] \wedge\left(\wedge^{n} T^{*} \boldsymbol{X}\right)$ and locally, $F_{\omega\left(\lambda, \mathfrak{G}(\bar{\Xi})_{V}\right)}=D_{H} M_{\omega\left(\lambda, \mathfrak{G}(\overline{\bar{\Xi}})_{V}\right)}$, with $M_{\omega\left(\lambda, \mathfrak{G}(\overline{\bar{\Xi}})_{V}\right)}$ :

$\left.: J_{4 s-1} \boldsymbol{Y}_{\zeta} \underset{\boldsymbol{X}}{\times} V J_{4 s-1} \mathcal{A}^{(r, k)}\right) \rightarrow \mathcal{C}_{2 s}^{*}\left[\mathcal{A}^{(r, k)}\right] \otimes \mathcal{C}_{2 s-1}^{*}\left[\mathcal{A}^{(r, k)}\right] \otimes \mathcal{C}_{0}^{*}\left[\mathcal{A}^{(r, k)}\right] \wedge\left({ }^{n-1} \wedge T^{*} \boldsymbol{X}\right)$.

Coordinate expressions for the morphisms $\beta\left(\lambda, \mathfrak{G}(\bar{\Xi})_{V}\right)$ and $M_{\omega\left(\lambda, \mathfrak{G}(\bar{\Xi})_{V}\right)}$ can be found by a backwards procedure (see e.g. 14]). In particular, $\beta\left(\lambda, \mathfrak{G}(\bar{\Xi})_{V}\right)$ is nothing but the Euler-Lagrange morphism associated with the new Lagrangian $\omega\left(\lambda, \mathfrak{G}(\bar{\Xi})_{V}\right)$ defined on the fibered manifold $J_{2 s} \boldsymbol{Y}_{\zeta} \underset{X}{\times} V J_{2 s} \mathcal{A}^{(r, k)} \rightarrow \boldsymbol{X}$. In particular, we get the following local decomposition of $\omega\left(\lambda, \mathfrak{G}(\bar{\Xi})_{V}\right)$ :

$$
\omega\left(\lambda, \mathfrak{G}(\bar{\Xi})_{V}\right)=\beta\left(\lambda, \mathfrak{G}(\bar{\Xi})_{V}\right)+D_{H} \tilde{\epsilon}\left(\lambda, \mathfrak{G}(\bar{\Xi})_{V}\right),
$$

where we put $\tilde{\epsilon}\left(\lambda, \mathfrak{G}(\bar{\Xi})_{V}\right) \equiv M_{\omega\left(\lambda, \mathfrak{G}(\bar{\Xi})_{V}\right)}$.

Definition 12 We call the global morphism $\beta\left(\lambda, \mathfrak{G}(\bar{\Xi})_{V}\right):=E_{\omega\left(\lambda, \mathfrak{G}(\bar{\Xi})_{V}\right)}$ the generalized Bianchi morphism associated with the Lagrangian $\lambda$. 
Remark 6 For any $(\bar{\Xi}, \xi) \in \mathcal{A}^{(r, k)}$, as a consequence of the gauge-natural invariance of the Lagrangian, by the Noether's First Theorem, the morphism $\beta\left(\lambda, \mathfrak{G}(\bar{\Xi})_{V}\right) \equiv \mathcal{E}_{n}\left(\omega\left(\lambda, \mathfrak{G}(\bar{\Xi})_{V}\right)\right)$ is locally identically vanishing. We stress that these are just local generalized Bianchi identities. In particular, we have locally $\omega\left(\lambda, \mathfrak{G}(\bar{\Xi})_{V}\right)=D_{H} \tilde{\epsilon}\left(\lambda, \mathfrak{G}(\bar{\Xi})_{V}\right)[2]$.

The form $\tilde{\epsilon}\left(\lambda, \mathfrak{G}(\bar{\Xi})_{V}\right) \equiv M_{\omega\left(\lambda, \mathfrak{G}(\bar{\Xi})_{V}\right)}$ is called a local reduced current. It vanishes along any critical section.

The problem of the general covariance of generalized Bianchi identities for field theories was posed by Anderson and Bergman already in 1951 (see [1]). Let now $\mathfrak{K}:=\operatorname{Ker}_{\mathcal{J}\left(\lambda, \mathfrak{G}(\bar{\Xi})_{V}\right)}$ be the kernel of the generalized gauge-natural morphism $\mathcal{J}\left(\lambda, \mathfrak{G}(\bar{\Xi})_{V}\right)$. As a consequence of Proposition 2 and of considerations above, we have the following covariant characterization of the kernel of generalized Bianchi morphism, the detailed proof of which will appear in 21.

Theorem 3 The generalized Bianchi morphism is globally vanishing if and only if $\delta_{\mathfrak{G}}^{2} \lambda \equiv \mathcal{J}\left(\lambda, \mathfrak{G}(\bar{\Xi})_{V}\right)=0$, i.e. if and only if $\mathfrak{G}(\bar{\Xi})_{V} \in \mathfrak{K}$.

The gauge-natural invariance of the variational principle in its whole enables us to solve the intrinsic indeterminacy in the conserved charges associated with gauge-natural symmetries of Lagrangian field theories (in [19], for example, the special case of the gravitational field coupled with fermionic matter is considered and the Kosmann lift is then invoked as an ad hoc choice to recover the well known expression of the Komar superpotential). By requiring the second variation to vanish, i.e. on the kernel of the Jacobi morphism, we express gaugenatural lits of infinitesimal principal automorphism in terms of the corresponding infinitesimal diffeomorphisms (their projections) on the basis manifolds (see Theorem 4 below). This is well known to be of great importance within the theory of Lie derivative of sections of a gauge-natural bundle and notably for the Lie derivative of spinors (see e.g. the review given in [19]).

Theorem 4 Let $\lambda \in \mathcal{V}_{s}^{n}$ be a gauge-natural invariant generalized Lagrangian and let $\mathfrak{G}(\bar{\Xi})$ be a gauge-natural lift of the principal infinitesimal automorphism $\bar{\Xi} \in \mathcal{A}^{r, k}$, i.e. a gauge-natural symmetry of $\lambda$. Then $\bar{\Xi} \in \mathcal{A}^{r, k}$ is related to its projection $\xi \in \mathcal{T}_{\boldsymbol{X}}$ by the condition

$(-1)^{|\boldsymbol{\sigma}|} D_{\boldsymbol{\sigma}}\left(D_{\boldsymbol{\mu}} \bar{\Xi}_{V}^{j}\left(\partial_{j}\left(\partial_{i}^{\boldsymbol{\mu}} \lambda\right)-\sum_{|\boldsymbol{\alpha}|=0}^{s-|\boldsymbol{\mu}|}(-1)^{|\boldsymbol{\mu}+\boldsymbol{\alpha}|} \frac{(\boldsymbol{\mu}+\boldsymbol{\alpha}) !}{\boldsymbol{\mu} ! \boldsymbol{\alpha} !} D_{\boldsymbol{\alpha}} \partial_{j}^{\boldsymbol{\alpha}}\left(\partial_{i}^{\boldsymbol{\mu}} \lambda\right)\right)\right)=0$.

Proof. We recall that given a vector field $j_{s} \hat{\Xi}: J_{s} \boldsymbol{Y}_{\zeta} \rightarrow T J_{r} \boldsymbol{Y}_{\zeta}$, the splitting (11) yields $j_{s} \hat{\Xi} \circ \pi_{s}^{s+1}=j_{s} \hat{\Xi}_{H}+j_{s} \hat{\Xi}_{V}$ where, if $j_{s} \hat{\Xi}=\hat{\Xi}^{\gamma} \partial_{\gamma}+\hat{\Xi}_{\alpha}^{i} \partial_{i}^{\alpha}$, then we have $j_{s} \hat{\Xi}_{H}=\hat{\Xi}^{\gamma} D_{\gamma}$ and $j_{s} \hat{\Xi}_{V}=D_{\boldsymbol{\alpha}}\left(\hat{\Xi}^{i}-y_{\gamma}^{i} \hat{\Xi}^{\gamma}\right) \partial_{i}^{\boldsymbol{\alpha}}$. Analogous considerations hold true of course also for the unique corresponding invariant vector field $j_{s} \bar{\Xi}$ on $W^{(r, k)} \boldsymbol{P}$. In particular, the condition $j_{s} \bar{\Xi}_{V}=D_{\boldsymbol{\alpha}}\left(\bar{\Xi}_{V}^{i}\right) \partial_{i}^{\boldsymbol{\alpha}} \in \mathfrak{K}$ implies, of course, that the components $\bar{\Xi}_{\boldsymbol{\alpha}}^{i}$ and $\bar{\Xi}^{\gamma}$ are not independent, but they are related in such a way that $j_{s} \bar{\Xi}_{V}=D_{\boldsymbol{\alpha}}\left(\hat{\Xi}^{i}-y_{\gamma}^{i} \hat{\Xi}^{\gamma}\right) \partial_{i}^{\boldsymbol{\alpha}}$ must be a solution of generalized gauge-natural Jacobi equations for the Lagrangian $\lambda$. 
Remark 7 For each $\bar{\Xi} \in \mathcal{A}^{(r, k)}$ such that $\bar{\Xi}_{V} \in \mathfrak{K}$, we have $\mathcal{L}_{j_{s} \bar{\Xi}_{H}} \omega(\lambda, \mathfrak{K})=0$; the latter is a naturality condition for the morphism $\omega(\lambda, \mathfrak{K})$ and says something on the Hamiltonian structure of the theory itself (see [12] for details).

The result above reflects in the theory of conserved currents and superpotentials for gauge-natural field theories, where the theory of Lie derivatives of sections of gauge-natural bundles finds one of its main application. In the following we shall refer to canonical globally defined objects (such as currents or corresponding superpotentials) by their explicit dependence on $\mathfrak{K}$.

Corollary 1 Let $\lambda \in \mathcal{V}_{s}^{n}$ be a gauge-natural Lagrangian and $j_{s} \hat{\Xi}_{V} \in \mathfrak{K}$ a gauge-natural symmetry of $\lambda$. Being $\beta(\lambda, \mathfrak{K}) \equiv 0$, we have, globally, $\omega(\lambda, \mathfrak{K})=$ $D_{H} \epsilon(\lambda, \mathfrak{K})$, then the following holds:

$$
D_{H}(\epsilon(\lambda, \mathfrak{K})-\tilde{\epsilon}(\lambda, \mathfrak{K})=0 .
$$

Eq. (12) is referred as a gauge-natural 'strong' conservation law for the global density $\epsilon(\lambda, \mathfrak{K})-\tilde{\epsilon}(\lambda, \mathfrak{K})$.

We can now state the following fundamental result about the existence and globality of canonical gauge-natural superpotentials in the framework of variational sequences.

Theorem 5 Let $\lambda \in \mathcal{V}_{s}^{n}$ be a gauge-natural Lagrangian and $\left(j_{s} \hat{\Xi}, \xi\right)$ a gaugenatural symmetry of $\lambda$. Then there exists a global sheaf morphism $\nu(\lambda, \mathfrak{K}) \in$ $\left(\mathcal{V}_{2 s-1}^{n-2}\right)_{\substack{\boldsymbol{Y}_{\boldsymbol{X}} \times \mathfrak{K} \\ \text { such that }}}$ such

$$
D_{H} \nu(\lambda, \mathfrak{K})=\epsilon(\lambda, \mathfrak{K})-\tilde{\epsilon}(\lambda, \mathfrak{K}) .
$$

Definition 13 We define the sheaf morphism $\nu(\lambda, \mathfrak{K})$ to be a canonical gaugenatural superpotential associated with $\lambda$.

Example 1 (Einstein-Yang-Mills theory) Let $\lambda \in \mathcal{V}_{s}^{n}$. It is known (see e.g. 14]) that

$$
\begin{gathered}
d_{V} \lambda=\left(d_{V} \lambda\right)_{i}^{\boldsymbol{\alpha}} \vartheta_{\boldsymbol{\alpha}}^{i} \wedge \omega, E_{d_{V} \lambda}=\mathcal{E}(\lambda)_{i} \vartheta^{i} \wedge \omega, p_{d_{V} \lambda}=p(\lambda)_{i}^{\boldsymbol{\alpha} \mu} \vartheta_{\boldsymbol{\alpha}}^{i} \wedge \omega_{\mu} \\
p(\lambda)_{i}^{\boldsymbol{\beta} \mu}=\left(d_{V} \lambda\right)_{i}^{\boldsymbol{\alpha}} \quad \boldsymbol{\beta}+\mu=\boldsymbol{\alpha},|\boldsymbol{\alpha}|=s \\
p(\lambda)_{i}^{\boldsymbol{\beta} \mu}=\left(d_{V} \lambda\right)_{i}^{\boldsymbol{\alpha}}-D_{\nu} p(\lambda)_{i}^{\boldsymbol{\alpha} \nu} \quad \boldsymbol{\beta}+\mu=\boldsymbol{\alpha},|\boldsymbol{\alpha}|=s-1 \\
\mathcal{E}(\lambda)_{i}^{\boldsymbol{\alpha}}=\left(d_{V} \lambda\right)_{i}^{\boldsymbol{\alpha}}-D_{\nu} p(\lambda)_{i}^{\boldsymbol{\alpha} \nu} \quad|\boldsymbol{\alpha}|=0 .
\end{gathered}
$$

Furthermore, $\mathcal{E}(\lambda)_{i}=\sum_{|\boldsymbol{\alpha}| \leq s}(-1)^{|\boldsymbol{\alpha}|} D_{\boldsymbol{\alpha}}\left(d_{V} \lambda\right)_{i}^{\boldsymbol{\alpha}}$.

Let $(\boldsymbol{P}, \boldsymbol{X}, \pi ; \boldsymbol{G})$ be a principal bundle, $g$ a metric on $\boldsymbol{X}, \mathfrak{k}$ an $a d$-invariant metric on $\boldsymbol{G}$. Let $\omega$ be a principal connection and $F$ its $\mathfrak{g}$-valued curvature 2-form. Let us now take the gauge-natural bundle $\boldsymbol{Y}=\operatorname{Lor}(\boldsymbol{X}) \underset{\boldsymbol{X}}{\times} \boldsymbol{C}$, where $\operatorname{Lor}(\boldsymbol{X})$ is the bundle of Lorentzian metrics over space-time $\boldsymbol{X}$ and $\boldsymbol{C}$ is the affine bundle of principal connections $\omega$ over $\boldsymbol{P}$. Local coordinates on $\boldsymbol{Y}$ are 
given by $x^{\mu}, g^{\mu \nu}, \omega_{\mu}^{i}$. Let us consider the gauge-natural Lagrangian $\lambda$ defined on the gauge-natural bundle $J_{2} \operatorname{Lor}(\boldsymbol{X}) \underset{\boldsymbol{X}}{\times} J_{1} \boldsymbol{C}$ :

$$
\lambda=\lambda_{H}\left(g^{\mu \nu}, R_{\mu \nu}\right)+\lambda_{Y M}\left(g^{\mu \nu}, F_{\mu \nu}^{i}\right),
$$

where $\lambda_{H}=-\frac{1}{2 \kappa} \sqrt{g} g^{\alpha \beta} R_{\alpha \beta}$ is the Einstein Lagrangian, $R_{\alpha \beta}$ is the Ricci tensor of the metric $g$ given by $R_{\alpha \beta}:=R_{\alpha \mu \beta}^{\mu}=D_{\mu} \gamma_{\alpha \beta}^{\mu}-D_{\beta} \gamma_{\alpha \mu}^{\mu}+\gamma_{\nu \mu}^{\mu} \gamma_{\alpha \beta}^{\nu}-\gamma_{\nu \beta}^{\mu} \gamma_{\alpha \mu}^{\nu}$, with $\gamma_{\nu \beta}^{\mu}=\frac{1}{2} g^{\mu \alpha}\left(D_{\nu} g_{\beta \alpha}-D_{\alpha} g_{\nu \beta}+D_{\beta} g_{\alpha \nu}\right)$ the Levi-Civita connection of $g$, $\sqrt{g}=\sqrt{\left|\operatorname{det}\left(g^{\mu \nu}\right)\right|}, \kappa$ is a constant and $\lambda_{Y M}\left(g_{\mu \nu}, F_{\mu \nu}^{i}\right)=-\frac{1}{4} \sqrt{g} F_{i}^{\lambda \gamma} F_{\lambda \gamma}^{i}$ is the Yang-Mills Lagrangian. Here $F_{i}^{\lambda \gamma}=\mathfrak{k}_{i j} g^{\lambda \alpha} g^{\gamma \beta} F_{\alpha \beta}^{j}$.

Notice that in this case $\boldsymbol{Y}_{\zeta}=J_{2} \operatorname{Lor}(\boldsymbol{X}) \underset{\boldsymbol{X}}{\times} J_{1} \boldsymbol{C}$ and the order of the gaugenatural bundle is $(r, k)=(3,2)$. An infinitesimal right-invariant automorphism of $\boldsymbol{P}$ is given in the split form:

$$
\Xi=\Xi_{h}+\Xi_{v}=\Xi^{\mu}\left(\partial_{\mu}+\omega_{\mu}^{i} \partial_{i}\right)+\left(\Xi^{i}-\omega_{\mu}^{i} \Xi^{\mu}\right) \partial_{i},
$$

where $\partial_{i}$ is a local basis of right-invariant vertical vector fields on $\boldsymbol{P}$. We shall respectively indicate by $\Xi_{v}$ and $\Xi_{h}$ the vertical and horizontal components of $\Xi$ with respect to the principal connection $\omega$; we shall write $\Xi_{v}^{i}=\Xi^{i}-\omega_{\mu}^{i} \Xi^{\mu}$. Notice that $\Xi_{v}^{i}$ is not equal to $\Xi_{V}^{i}$, but they are related in a clear and simple way. We get $\epsilon^{\sigma}\left(\lambda, \Xi_{h}\right)=\epsilon^{\sigma}\left(\lambda_{H}, \Xi_{h}\right)+\epsilon^{\sigma}\left(\lambda_{Y M}, \Xi_{h}\right)$, where

$$
\epsilon^{\sigma}\left(\lambda_{H}, \Xi_{h}\right)=\frac{1}{\kappa} \sqrt{g}\left(R_{\beta}^{\sigma}-R g_{\beta}^{\sigma}\right) \Xi^{\beta}+\nabla_{\mu}\left[\frac{\sqrt{g}}{2 \kappa}\left(\nabla^{\sigma} \Xi^{\mu}-\nabla^{\mu} \Xi^{\sigma}\right)\right] .
$$

and

$\epsilon^{\sigma}\left(\lambda_{Y M}, \Xi_{h}\right)=\left(2 p_{i}^{\mu \sigma} £_{\Xi_{h}} \omega_{\mu}^{i}-\lambda_{Y M} \Xi^{\sigma}\right)=-\sqrt{g}\left(F_{i}^{\mu \sigma} F_{\mu \nu}^{i}-\frac{1}{4} F_{i}^{\mu \rho} F_{\mu \rho}^{i} \delta_{\nu}^{\sigma}\right) \Xi^{\nu}$.

$R$ is the scalar curvature and $p_{i}^{\mu \nu}=-\frac{\sqrt{g}}{2} F_{i}^{\mu \nu}$. Here and in the sequel $\nabla_{\mu}$ denotes the covariant metric derivative with respect to $g$.

A "horizontal" superpotential is given by: $\nu_{h}^{\sigma \mu}=\frac{\sqrt{g}}{4 \kappa}\left(\nabla^{\sigma} \Xi^{\mu}-\nabla^{\mu} \Xi^{\sigma}\right)$, which is essentially the Komar superpotential [2].

Furthermore, we have $\epsilon^{\sigma}\left(\lambda_{Y M}, \Xi_{v}\right)=-2 p_{i}^{\mu \sigma} \nabla_{\mu} \Xi_{v}^{i}=-\nabla_{\mu}\left(-2 p_{i}^{\mu \sigma} \Xi_{v}^{i}\right)+$ $2 \nabla_{\mu} p_{i}^{\mu \sigma} \Xi_{v}^{i}$. Then there exists a "vertical" superpotential, given by:

$$
\nu_{v}^{\mu \sigma}=p_{i}^{\mu \sigma} \Xi_{v}^{i}=-\frac{\sqrt{g}}{2} F_{i}^{\mu \sigma} \Xi_{v}^{i} .
$$

From Theorem 4 we deduce that also the components $\Xi_{v}^{i}$ - when $\Xi_{V}$ is an element of the kernel of the gauge-natural generalized Jacobi morphism - can be expressed invariantly in terms of the components $\Xi^{\mu}$ of the projection of $\Xi_{v}$ :

$$
(-1)^{|\boldsymbol{\sigma}|} D_{\boldsymbol{\sigma}}\left(D_{\boldsymbol{\mu}}\left(\hat{\Xi}_{v}^{i}+\left(\hat{\omega}_{\gamma}^{i}-y_{\gamma}^{i}\right) \hat{\Xi}^{\gamma}\right) \psi_{j i}^{\boldsymbol{\mu}}\right)=0,
$$

where $\psi_{j i}^{\boldsymbol{\mu}}=\left(\partial_{j}\left(\partial_{i}^{\boldsymbol{\mu}} \lambda\right)-\sum_{|\boldsymbol{\alpha}|=0}^{s-|\boldsymbol{\mu}|}(-1)^{|\boldsymbol{\mu}+\boldsymbol{\alpha}|} \frac{(\boldsymbol{\mu}+\boldsymbol{\alpha}) !}{\boldsymbol{\mu} ! \boldsymbol{\alpha} !} D_{\boldsymbol{\alpha}} \partial_{j}^{\boldsymbol{\alpha}}\left(\partial_{i}^{\boldsymbol{\mu}} \lambda\right)\right)$ and $\hat{\omega}$ is the gauge natural prolongation of $\omega$ (see []). When such a dependence is expressed explicitly, we write $\nu_{v}^{\mu \sigma}=\nu_{v}^{\mu \sigma}(\lambda, \mathfrak{K})$. 
Equivalently, given $\Xi_{v}^{i}$ as above, Theorem 4 says us also that some constraint on the connection $\omega$ is there. It is maybe noteworthy that $\lambda$ is the total Lagrangian (13).

Acknowledgments. The authors wish to thank I. Kolár and D. Krupka for many interesting discussions and the unknown referee for useful remarks which lead to improve the text.

\section{References}

[1] J.L. Anderson, P.G. Bergmann: Constraints in Covariant Field Theories, Phys. Rev. 83 (5) (1951) 1018-1025.

[2] P.G. Bergman: Non-Linear Field Theories, Phys. Rev. 75 (4) (1949) 680-685. P.G. Bergman: Conservation Laws in General relativity as the Generators of Coordinate Transformations, Phys. Rev. 112 (1) (1958) 287-289. J.N. GoldBerg: Conservation laws in general relativity. Phys. Rev. (2) 111 (1958) 315-320. A. Komar: Covariant Conservation Laws in General Relativity, Phys. Rev. 113 (1959) (3) 934-936.

[3] C. Caratheodory: Calculus of variations and partial differential equations of the first order, Chelsea Publ. Co., New York, 1982, p. 262.

[4] P.T. Chruściel: On the relation between the Einstein and the Komar expressions for the energy of the gravitational field, Ann. Inst. Henri Poincaré 42 (3) (1985) 267-282. B. Julia, S. Silva: Currents and superpotentials in classical gauge theories. II. Global Aspects and the example of affine gravity, Class. Quant. Grav., 17 (22) (2000) 47334743. J. KATZ: A note on Komar's anomalous factor, Classical Quantum Gravity 2 (3) (1985) 423-425.

[5] D.J. EcK: Gauge-natural bundles and generalized gauge theories, Mem. Amer. Math. Soc. 247 (1981) 1-48.

[6] L. Fatibene, M. Francaviglia, M. Palese: Conservation laws and variational sequences in gauge-natural theories, Math. Proc. Camb. Phil. Soc. 130 (2001) 555-569.

[7] M. Ferraris, M. Francaviglia: The Lagrangian Approach to Conserved Quantities in General Relativity, in: Mechanics, Analysis and Geometry: 200 Years after Lagrange; M. Francaviglia ed.; Elsevier Science Publishers B. V. (Amsterdam, 1991), 451-488.

[8] M. Francaviglia, M. Palese: Second Order Variations in Variational Sequences, Steps in differential geometry (Debrecen, 2000) Inst. Math. Inform. Debrecen, Hungary (2001) $119-130$.

[9] M. Francaviglia, M. Palese: Generalized Jacobi morphisms in variational sequences, in Proc. XXI Winter School Geometry and Physics, Srni 2001 Rend. Circ. Matem. di Palermo. Serie II, Suppl. 69 (2002) 195-208.

[10] M. Francaviglia, M. Palese, R. Vitolo: Symmetries in Finite Order Variational Sequences, Czech. Math. J. 52(127) (2002) 197-213.

[11] M. Francaviglia, M. Palese, R. Vitolo: The Hessian and Jacobi Morphisms for Higher Order Calculus of Variations, Diff. Geom. Appl. 22 (1) (2005) 105-120.

[12] M. Francaviglia, M. Palese, E. Winterroth: Generalized Bianchi identities in gauge-natural field theories and the curvature of variational principles, preprint. 
[13] M. Godina, P. Matteucci: Reductive G-structures and Lie derivatives, J. Geom. Phys. 47 (1) (2003) 66-86.

[14] I. KolÁř: A Geometrical Version of the Higher Order Hamilton Formalism in Fibred Manifolds, J. Geom. Phys., 1 (2) (1984) 127-137.

[15] I. Kolár̆, P.W. Michor, J. Slovák: Natural Operations in Differential Geometry, (Springer-Verlag, N.Y., 1993).

[16] I. KolÁř, R. Vitolo: On the Helmholtz operator for Euler morphisms, Math. Proc. Cambridge Phil. Soc., 135 (2) (2003) 277-290.

[17] D. Krupka: Variational Sequences on Finite Order Jet Spaces, Proc. Diff. Geom. and its Appl. (Brno, 1989); J. Janyška, D. Krupka eds.; World Scientific (Singapore, 1990) 236-254.

[18] L. Mangiarotti, M. Modugno: Fibered Spaces, Jet Spaces and Connections for Field Theories, in Proc. Int. Meet. on Geom. and Phys.; M. Modugno ed.; Pitagora Editrice (Bologna, 1983) 135-165.

[19] P. Matteucci: Einstein-Dirac theory on gauge-natural bundles, Rep. Math. Phys. 52 (1) (2003) 115-139.

[20] E. NÖтher: Invariante Variationsprobleme, Nachr. Ges. Wiss. Gött., Math. Phys. Kl. II (1918) 235-257.

[21] M. Palese, E. Winterroth: Global Generalized Bianchi Identities for Invariant Variational Problems on Gauge-natural Bundles, to appear in Arch. Math. (Brno).

[22] H. Rund: The Hamilton-Jacobi theory in the calculus of variations, D. Van Nostrand Company LTD (London 1966) p. 127.

[23] D.J. SAunders: The Geometry of Jet Bundles, Cambridge Univ. Press (Cambridge, 1989).

[24] A. Trautman: Conservation laws in general relativity. in Gravitation: An introduction to current research pp. 169-198 Wiley, New York (1962). A. Trautman: Noether equations and conservation laws, Comm. Math. Phys. 6 (1967) 248-261.

[25] R. Vitolo: Finite Order Lagrangian Bicomplexes, Math. Proc. Camb. Phil. Soc. 125 (1) (1999) 321-333. 